\title{
Prevalencia de caries dental según el índice CPOD en pacientes adultos chacchadores de hojas de coca.
}

\author{
PREVALENCE OF DENTAL CARIES AS DMFT INDEX IN ADULT PATIENTS CHACCHADORES \\ COCA LEAVES.
}

Cinthya Gissella Torres Portuguez ${ }^{1}$, Melvin Cisneros del Aguila ${ }^{2}$

\begin{abstract}
${ }^{1}$ Cirujano Dentista Egresada de la Escuela de Estomatología de la Universidad Alas Peruanas, Lima, Perú.
2 Cirujano Dentista, Maestro en Investigación y Docencia Universitaria, Doctor en Odontología, Coordinador de la Asignatura de Prótesis Parcial Removible, Escuela de Estomatología de la Universidad Alas Peruanas, Lima, Perú.

Correspondencia:

Melvin Cisneros del Aguila

Jr. Carlos de los Heros 750 Pueblo Libre, Lima, Perú.

Teléfono: 998672762

Correo electrónico: cisnerosmelvin@hotmail.com
\end{abstract}

\section{RESUMEN}

Objetivo: Se buscó determinar la prevalencia de caries dental, actitudes en el hábito del chacchado de coca y el índice CPOD en una población de adultos de 20 a 60 años. Material y métodos: La muestra correspondió a 365 chacchadores de coca; mediante un examen bucal previo se hizo una pre-selección de la población para incluirlos o excluirlos del estudio; los incluidos llenaron un cuestionario y se les realizó el examen odontológico correspondiente. Resultados: La mayor frecuencia de chacchado fue de una vez al día (34\%). El complemento más utilizado fue la cal (60\%). La prevalencia de caries fue de (98\%). El índice CPOD fue de 7,56. Conclusiónes: La cal y mishquina fueron complementos preferidos por el chachador; a mayor edad mayor cantidad de piezas perdidas y con indicación de exodoncia. La prevalencia de caries fue bastante elevada y el índice CPOD correspondió a un nivel de severidad muy alto.

PALABRAS CLAVE: Chacchado de coca, prevalencia de caries, índice CPOD. 


\section{SUMMARY}

Objective: We sought to determine the prevalence of dental caries, positioned between the habits of coca chewers and the DMFT index in a population of adults aged 20 to 60 years. Material and methods: The sample consisted of 365 coca chewers determined through preliminary oral examination and pre-selection of the population to include or exclude in the study. Those included completed a questionnaire and underwent the appropriate dental examination. Results: The highest frequency of chewing was once daily ( $34 \%$ ). Lime was used as a complement ( $60 \%$ ). The prevalence of caries was (98\%). The DMFT index was 7.56. Conclusions: Lime and mishquina were the prefered complements for the chewers; age corresponded with an increase in already missing teeth including more needing extraction. The prevalence of cavities was quite high and the DMFT corresponds to a higher level of severity.

KEY WORDS: Coca chewers, caries prevalence, DMFT index.

\section{INTRODUCCIÓN}

La coca (Erytroxylum coca) es un arbusto que crece en las zonas de selva alta de Bolivia, Colombia y Perú, aproximadamente entre los 500 y 1000 metros de altura; la variedad Erytroxylum novagranatense, es la que crece en Perú. Según Aibar (2006) en condiciones óptimas puede pervivir hasta los cien años. Forma parte de las costumbres, creencias, cultos, mitos y de la medicina peruana, Dominic citado por Jerí $(1980)(1,2)$.

Durante la colonia su uso fue incentivado como medio de explotación laboral hacia los indígenas y al mismo tiempo fue usada en transacciones comerciales con otros países, como lo relata Marroquín citado por Jerí (1980) y Carrillo (1990) (2, 3). 
Sus componentes son el salicilato de metilo, además de flavonoides y taninos, Bruneton (2001) menciona que la cocaína (metilbenzoilecgonina) representa el 30 a 50 \% de su composición. Además le atribuyen grandiosas propiedades alimenticias, en este aspecto Ramos (2005) destaca los altos contenidos proteicos. Duke, citado por Veliz, Velezmoro, Monte y Jáuregui (1989), menciona que contiene vitaminas A, B, C y 14 alcaloides que pertenecen a la serie del propano (4 - 6).

A lo largo de la historia, la masticación de la hoja de coca ha sido considerada como dañino para la salud, hasta que la Organización de las Naciones Unidas (ONU) en mayo de 1950 aclara que no constituye una toxicomanía, sino un hábito, además concluyó tajantemente que el "indio" no mastica la coca, sino "chaccha". El hábito de la masticación es conocido con distintos nombres; de acuerdo con Cabieses (2001), los vocablos indígenas para este acto son "chacchar", "picchar" y "acullicar", comprende todo un ritual mágico andino, se forma un bolo con la saliva ayudado por una sustancia alcalina Ilamada "Ilipta" o "troca". En este estudio utilizaremos el primero por considerarlo más original. De acuerdo con Gutierrez citado por la ONU (1950), la cantidad de hojas utilizado tiene un peso promedio aproximado de 30 gramos. Sobre este mismo tema Castro de la Mata citado por Jerí (1980), el chacchado lo realizan durante el trabajo o una caminata $(7,8,2)$.

La hoja de coca tiene diversos usos medicinales, según Aibar (2006) incentiva la diminución de la grasa corporal mediante la acción de la cocaína al transformarse en ecgonina; Díaz et al (2007) refieren que el extracto de E. coca lam var. Coca, tiene un buen efecto coagulante $(1,9)$.

La boca es el receptáculo de diversidad de alimentos; en particular durante el chacchado se llevan a ella hojas de coca y sustancias alcalinas, constituye también el 
hogar de distintos microorganismos muchos de ellos cariogénicos. En este aspecto, Higashida (2009), refiere que el Streptococcus mutans es el de mayor potencial cariogénico, aunque también son importantes $S$. salivarius, $S$. milleri, $S$. sanguis, $S$. mitis, S. intermedious, Lactobacillus acidophilus, L. casei, Actinomyces viscosus y A. naes/undii, entre otros (10).

La biopelícula o placa dental adherida a las superficies dentarias Según Marcantoni citado por Barrancos y Barrancos (2009), es una entidad bacteriana con actividad enzimática y que mediante su acción bioquímica y metabólica, es el principal agente etiológico de la caries dental. A mayor ingesta de carbohidratos, mayor posibilidad de caries, Nuñez y García (2010), Cawson y Odell (2009), coinciden en que la colonización por bacterias cariógenas, sobre todo por S. mutans, depende en gran medida del contenido de sacarosa en la dieta $(11,12,13)$

Existen pocos estudios, algunos de ellos significativos referentes a la relación del chacchado de hojas de coca y la caries dental. En este aspecto Ungaro (1972) encontró una alta prevalencia de caries dental y abrasión dentaria. Flores (1997) concluyó que no existe relación significativa entre los índice CPOD y CPOS con la presencia, tiempo y frecuencia del hábito $(14,15)$

En 1998 Granados reportó que el índice de caries dental CPO es menor en personas con el hábito $(9,3 \pm 7,2)$, que en aquellos que no presentan el hábito $(13,2 \pm$ 6,8). Molina (1988) encontró una prevalencia de caries de 9,67\% para los masticadores de hoja de coca; concluye que la frecuencia de caries dental en los chacchadores, es baja. Zárate (1999) refiere que los chacchadores practican el hábito masticándola con una mezcla de la sustancia alcalina "llipta". Ramos (2008) en un estudio epidemiológico, 
determino que el CPOD de los sujetos con el hábito es 11,58 y de los sin el hábito es de $17,3(16,17,18,19)$.

En las serranías de Perú y Bolivia especialmente y de Colombia y Chile en menor escala, la práctica del chacchado de hojas de coca es habitual y está considerado como parte de una rutina diaria. En estas zonas, los servicios de salud son escasos y por lo tanto los problemas en este ámbito son mayores. En cuando a la salud bucal, estos aspectos repercuten negativamente generando una mayor prevalencia de caries dental y enfermedad periodontal.

En este estudio se buscó evaluar la frecuencia diaria de bolos utilizados por los chacchadores de hoja de coca, identificar el complemento más utilizado para el chacchado, determinar el porcentaje de piezas cariadas, con indicación de exodoncia, obturadas y perdidas por caries en relación al grupo etáreo y en el total de la muestra, también determinar el índice CPOD de acuerdo a la edad y en el total de la muestra.

Enfocado de este punto de vista, este estudio se trazó como objetivo general determinar la prevalencia de caries dental en una población de adultos de 20 a 60 años chacchadores de hojas de coca.

\section{MATERIAL Y MÉTODOS}

Este observacional, prospectivo, transversal y descriptivo, involucró a una población de 4170 habitantes del distrito de José Manuel Quiroz, Cajamarca, Perú; cuya muestra no probabilística intencional correspondió a 365 individuos de ambos sexos comprendidos entre los 20 y 60 años de edad. En el estudio se incluyeron a sujetos chacchadores de hoja de coca, a edéntulos parciales y a los portadores de prótesis parcial fija, prótesis parcial removible y prótesis completa unimaxilar; se excluyeron a los sujetos con limitación de la apertura bucal y con enfermedades psiquiátricas y neurológicas. A todos 
los sujetos participantes en el estudio se les hizo conocer y firmar el documento de consentimiento informado. Los datos se recolectaron utilizando: instrumental de rutina para el examen estomatológico, una ficha de clasificación, historia clínica estomatológica y un cuestionario. En cada uno de los integrantes de la muestra y utilizando el índice CPOD, se evaluó la prevalencia de caries dental de acuerdo a la edad, además la condición y actitud de los chacchadores de hoja de coca.

El procedimiento de selección de la muestra se inició con el llenado de la ficha de evaluación clasificando a los sujetos de acuerdo a los criterios de inclusión y exclusión. El segundo paso correspondió al llenado del cuestionario poniendo especial interés en que sean contestadas todas las interrogantes planteadas; a cada cuestionario se le asignó un número para efectos de control.

El tercer momento correspondió a la realización de la anamnesis y el examen clínico estomatológico respetivo, utilizando para esto un espejo bucal sin aumento y un explorador; los datos recabados se anotaron en la historia clínica diseñada exclusivamente para este estudio. Terminado el examen clínico, a cada historia clínica se le asignó un número correlativo para efectos de control, coincidiendo este número con el del cuestionario. Los datos fueron procesados utilizando estadística descriptiva.

\section{RESULTADOS}

De los 365 participantes en el estudio, 356 (98\%) presentó caries entre leve y moderada, nueve (2\%) estuvieron libres de lesión cariosa. En referencia a la frecuencia diaria de chacchado de hojas de coca, se encontró que el porcentaje mayor de personas que chacchan, es de 1 vez al día (v/d) que correspondió a 124 (34\%); los que chacchan 2 v/d abarcó a 107 (29,3\%); a su vez representaron un número intermedio 
entre mayor y menor los que practican el hábito de manera ocasional aglutinando estos a 67 individuos (18,4\%); en menores proporciones estuvieron los que chacchan 3 v/d 58 y cuatro veces diarias $(2,5 \%)$ (Tabla 1$)$.

En relación al complemento utilizado para el chacchado de hojas de coca, dentro de los seis elementos empleados para tal fin, la cal, fue la más utilizada, 219 individuos (60\%), constituyendo así el complemento preferido para realizar el chacchado; la combinación de cal y mishquina $78(21 \%)$, fue la segunda en preferencia; en menores proporciones utilizaron cal/ceniza 32 chacchadores (9\%); 19 individuos (5\%), realizaron el chacchado unas veces utilizando cal, otras veces sola, es decir, de forma intermitente; en menor cantidad, utilizaron cal/azúcar 13 individuos (4\%) y, sola sin ningún complemento 4 individuos (1\%) (Figura 1).

Referente a la distribución de piezas cariadas en el total de la muestra de acuerdo a grupo etáreo, los resultados nos muestran que el grupo más vulnerable corresponde al de 30-39 años con 481 piezas cariadas (34,5\%); con cantidades muy similares están los grupos de 20-29 y 50-60 años, en ellos se encontraron 330 (23,7\%) y $320(23 \%)$ piezas cariadas respectivamente; el grupo menos vulnerable correspondió al de 40-49 años con 262 (18,8\%) piezas cariadas (Tabla 2).

En relación a las piezas perdidas de acuerdo a grupo etáreo, en el de 50-60 años la ausencia de piezas corresponde a $658(55,2 \%)$; en orden decreciente está el grupo de 40-49 años con 269 piezas perdidas (22,6\%); en el grupo de 30-39 años se hallaron 197 piezas ausentes (16,5\%); el grupo etáreo que menos piezas ausentes presenta es el de 20-29 años con 68 piezas perdidas (5,7\%) (Tabla 3$)$. 
En referencia a la distribución de piezas obturadas de acuerdo a grupo etáreo, el porcentaje más alto corresponde al grupo de 30-39 años con 69 piezas obturadas $(39,4 \%)$; en segunda instancia encontramos al grupo de 20-29 años con 46 piezas obturadas (26,3\%); los grupos etáreos con menos piezas obturadas corresponden al de 50-60 años que muestra 34 piezas obturadas (19,4\%); y el de 40-49 años con 26 piezas obturadas (14,9\%) (Tabla 4).

En lo concerniente a la distribución de piezas carias con indicación de exodoncia de acuerdo a grupo etáreo, se encontró que en el grupo de 50-60 años las piezas cariadas con indicción de exodoncia alcanza el número más alto, es de 116 (51,8\%); En orden decreciente está el grupo de $40-49$ años con 59 piezas (26,3\%); en el grupo de 30-39 años se hallaron 38 piezas con indicación de exodoncia (17\%); el grupo etáreo que menos piezas cariadas con indicación de exodoncia es el de 20-29 años con 11 piezas $(4,9 \%)$ (Tabla 5$)$.

En relación a la distribución de piezas cariadas, perdidas, obturadas y cariadas con indicación de exodoncia en el total de la muestra, se halló que las cantidades más altas corresponden a piezas perdidas $1192(44,9 \%)$ y piezas cariadas $1063(40,1 \%)$; las piezas cariadas con indicación de exodoncia corresponden a $224(8,4 \%)$; y aún en menor cantidad están las piezas obturadas 175 (6,6\%) (Figura 2).

En lo concerniente al índice CPOD de acuerdo a grupo etáreo, encontramos que el de más alto índice CPOD fue el de 50-60 años con 10,22; en orden decreciente se encontró el grupo etáreo de 40-49 años con un índice de 7,96; el de 30-39 años presentó un índice de 6,38; el grupo etáreo cuyo índice CPOD fue el más bajo, 
correspondió al de 20-29 años con 5,62. El índice CPOD del total de la muestra 365 individuos, correspondió a 7,56 (Tabla 6).

\section{DISCUSIÓN}

Este estudio tuvo la finalidad de determinar la prevalencia de caries dental en una muestra conformada por chacchadores de hojas de coca, del mismo modo se identificó el índice CPOD. La prevalencia de caries encontrada en este estudio es bastante elevada, correspondiendo está al (98\%), la muestra en la cual se realizó el estudio fue de 365 individuos, la misma que avala su significancia y nos lleva a la conclusión que la deficiente higiene bucal y la falta de servicios de salud odontológica nos muestra esta realidad; estos datos son similares a los encontrados por Ungaro (1972) en una muestra también bastante significativa y los mencionados por Ramos (2008), este último en una muestra menor. Además mencionaremos que los datos sobre caries de nuestro estudio son disímiles a los de Molina (1988) en una muestra de menor cuantía $(14,19,17)$.

En lo referente a los chacchadores, se investigó la frecuencia con que realizan el hábito y el complemento que utilizan para realizar este acto rutinario del hombre del ande peruano. De acuerdo con la literatura revisada, el chacchado de hojas de coca es un hábito milenario propio de nuestras serranías y de otras como Bolivia, parte de Chile y Argentina, se conoce que este acto no convierte al individuo inmerso en un adicto, nuestros resultados corroboran esto porque la frecuencia del chacchado es en una gran mayoría entre una vez al día (34\%), dos veces al día (29\%) y ocasionalmente (18,4\%), esto corresponde al $(81,4 \%)$ del total de la muestra; significa esto que el chacchador realiza el hábito como una costumbre tradicional y en el entendimiento de que le va a ser beneficioso para su salud y como energizante para coadyuvar en su estado de 
ánimo y físico en la realización de sus labores cotidianas. De acuerdo con esto asumimos que el chacchador no utiliza las hojas de coca para beneficiarse única y exclusivamente del efecto causado por su principal alcaloide "la cocaína" por lo tanto y de acuerdo con los datos encontrados, el chacchador no debe ser considerado como un drogadicto, porque no está sujeto al efecto de adicción que tienen los diferentes estupefacientes. Esta apreciación asumida concuerda con el informe de la comisión de estudio de las hojas de coca realizado por la ONU (1950) donde menciona que el chacchado no representa una toxicomanía sino un hábito (7).

El chacchado de la hoja de coca hecho por el poblador andino, lo realiza acompañado de una sustancia alcalina y la mayor parte lo hacen utilizando cal sola, además de esta acompañada con mishquina, estos dos elementos utilizados como complementos representan el $(81 \%)$ del total de complementos utilizados para este hábito, datos que coinciden con los mencionados por Zárate (1999), La ONU (1950) y Cabieses $(2001)(18,7,8)$.

Analizando los porcentaje de piezas cariadas, con indicación de exodoncia, obturadas y perdidas por caries de acuerdo a grupo etáreo encontramos que las piezas perdidas marcan una tendencia ascendente, esta va de 5,75 en el grupo de 20-29 años hasta el (55,2\%) en el grupo de 50-60 años; significa esto que por acción de la caries dental, las piezas dentarias en la población más adulta se han ido perdiendo, demostrando a la vez que en estos grupos poblacionales el aspecto preventivo hacia las enfermedades prevalentes de la cavidad oral es pobre. Del mismo modo las piezas cariadas con indicación de exodoncia, marcan la misma tendencia, es decir, una cantidad menor en el grupo etáreo de $20-29$ años (4,9\%) y en el extremo más alto el grupo etáreo de mayor edad 50-60 años con (51,8\%). Estos datos son el fiel reflejo de 
la precaria higiene bucal de los individuos chacchadores de hoja de coca que conforme avanzan en edad esta es más deficiente.

Es de significancia resaltar que del disgregado del CPOD del total de la muestra, en nuestro estudio arroja que las piezas cariadas y perdidas corresponden al (85\%) demostrando una vez más que esto se debe a una baja cultura en relación a la prevención de enfermedades prevalentes de la cavidad bucal.

En nuestro estudio se hizo el análisis del índice CPOD de acuerdo a grupo etáreo, los resultados igual que las piezas perdidas y las cariadas con indicación de exodoncia, muestran una tendencia ascendente de menor a mayor edad, por lo tanto el nivel de severidad en el grupo etáreo de 20-29 años es alto 5,62; y muy alto 10,22 en el grupo de 50-60 años.

El índice CPOD total de la muestra estudiada corresponde a 7,56, por lo tanto se concluye que en cuanto a criterios epidemiológicos el nivel de severidad del índice CPOD en chacchadores de hoja de coca es muy alto. En otros estudios como el de Granados (1998), el índice correspondió a 9,3 \pm 7,2 muy cercano a nuestros resultados. Asimismo las investigaciones realizadas por Ramos (2008) y Flores (1997), nos muestran también niveles de severidad muy altos 11,58 y 14,5 respectivamente. Se concluye que cuanta más adulta es la población de chacchadores de hoja de coca, es mayor el índice CPOD. $(16,5,15)$.

\section{REFERENCIAS BIBLIOGRÁFICAS}

1.- Aibar M. Los efectos del consumo de hoja de coca. Revista Peruana de Obstetricia y Enfermería 2006; 1 (2): 71-80. 
2.- Jerí F. Cocaína: Actas del seminario interamericano. Lima: Editorial Pacific Press; 1980.

3.- Carrillo J. Cocaína: Problemas y soluciones andinos. Lima: Editorial Nova Print S.R.L.; 1990.

4.- Bruneton J. Farmacognosia. Fitoquímica. Plantas medicinales. Zaragoza: Editorial Acribia S. A.; 2001.

5.- Ramos R. Fraccionamiento químico de la hoja de coca y obtención de un producto rico en proteínas. Revista de la Sociedad Química del Perú 2005; 71 (1): 3-11.

6.- Veliz A, Velezmoro V, Monte G y Jáuregui P. Estudio de las condiciones de salud bucal de los nativos masticadores de coca del ande peruano. Revista de la Academia de Estomatología del Perú 1989; 53 (1): 35-46.

7.- Naciones Unidas. Informe de la comisión de estudio de las hojas de coca. Lima: editorial ONU; 1950.

8.- Cabieses F. Más sobre la coca. Lima: Editorial H. Comercial S.A.C; 2001.

9.- Díaz A, Pérez L, Castro A, Chein S, Sánchez J, Tenorio J, et al. Efecto coagulante de dos variedades de hoja de coca en muestras de sangre de ratas albinas. Odontología San Marquina 2007; 10 (1): 7-9.

10.- Higashida B. Odontología preventiva. México, DF: Litográfica ingramex; 2009.

11.- Barrancos J, Barrancos P. Operatoria dental, integración clínica. Argentina: Editorial médica panamericana S. A; 2009.

12.- Núñez D. P, García L. Bioquímica de la caries dental. Revhabanciencméd 2010; 9 (2): 156-166. [Revista en Internet]. [Citado 2013 Ene 09]. Disponible en: http://scielo.sld.cu/scielo.php?script=sci_arttext\&pid=S1729519X2010000200004\&lng=e S. 
13.- Cawson R, Odell E. Cawson fundamentos de medicina y patología oral. España: Elsevier España S.L; 2009.

14. Ungaro M. Patología oral en masticadores de hoja de coca. [Tesis para optar el grado de Doctor en Medicina]. Lima: Universidad Peruana Cayetano Heredia; 1972.

15.- Flores F. Prevalencia de caries, enfermedad periodontal y desgaste dentario en sujetos entre 40 y 70 años de edad según hábitos de masticación de hojas de coca en la comunidad de Quircan, distrito de Mosca, Provincia de Ambo, Departamento Huánuco. [Tesis para optar el título profesional de Cirujano Dentista]. Lima: Universidad Peruana Cayetano Heredia; 1997.

16.- Granados A. Abrasión y caries dental en chacchadores de coca y no chacchadores de coca en el distrito de la Encañada Cajamarca. [Tesis para optar el título profesional de Cirujano Dentista]. Lima: Universidad San Martín de Porres; 1998.

17.- Molina M. Frecuencia de caries dental y abrasión dentaria en pacientes masticadores de coca. Distrito de San Pedro-Provincia de Lucanas Ayacucho. [Tesis para optar el título profesional de Cirujano Dentista]. Lima: USMP; 1998.

18.- Zárate R. Estudio gingival en personas masticadoras de hoja de coca del distrito de Aco, Provincia de Concepción-Junín. [Tesis para optar el título profesional de Cirujano Dentista]. Lima: Universidad San Martín de Porres; 1999.

19.- Ramos E. Efectividad de la masticación de la hoja de coca en la prevención de la caries dental en el centro poblado de San Juan de la Libertad Huasahuasi Tarma. [Tesis para optar el título profesional de Cirujano Dentista]. Lima: Universidad Nacional Federico Villarreal; 2008. 
Tabla 1. Frecuencia diaria de chacchado de hojas de coca por los integrantes de la muestra

\begin{tabular}{|c|c|c|c|c|c|c|c|c|}
\hline $\begin{array}{l}\text { FRECUENCIA } \\
\text { DE BOLOS }\end{array}$ & $\begin{array}{l}\text { Una } \\
\text { diaria }\end{array}$ & $\%$ & $\begin{array}{l}\text { Dos } \\
\text { diarias \% }\end{array}$ & $\begin{array}{l}\text { Tres } \\
\text { diarias \% }\end{array}$ & $\begin{array}{l}\text { Cuatro } \\
\text { diarias }\end{array}$ & $\%$ & Ocasional \% & TOTAL \% \\
\hline & 124 & $34,0 \%$ & $29,3 \%$ & $15,9 \%$ & 9 & $2,5 \%$ & $18,4 \%$ & $100 \%$ \\
\hline
\end{tabular}

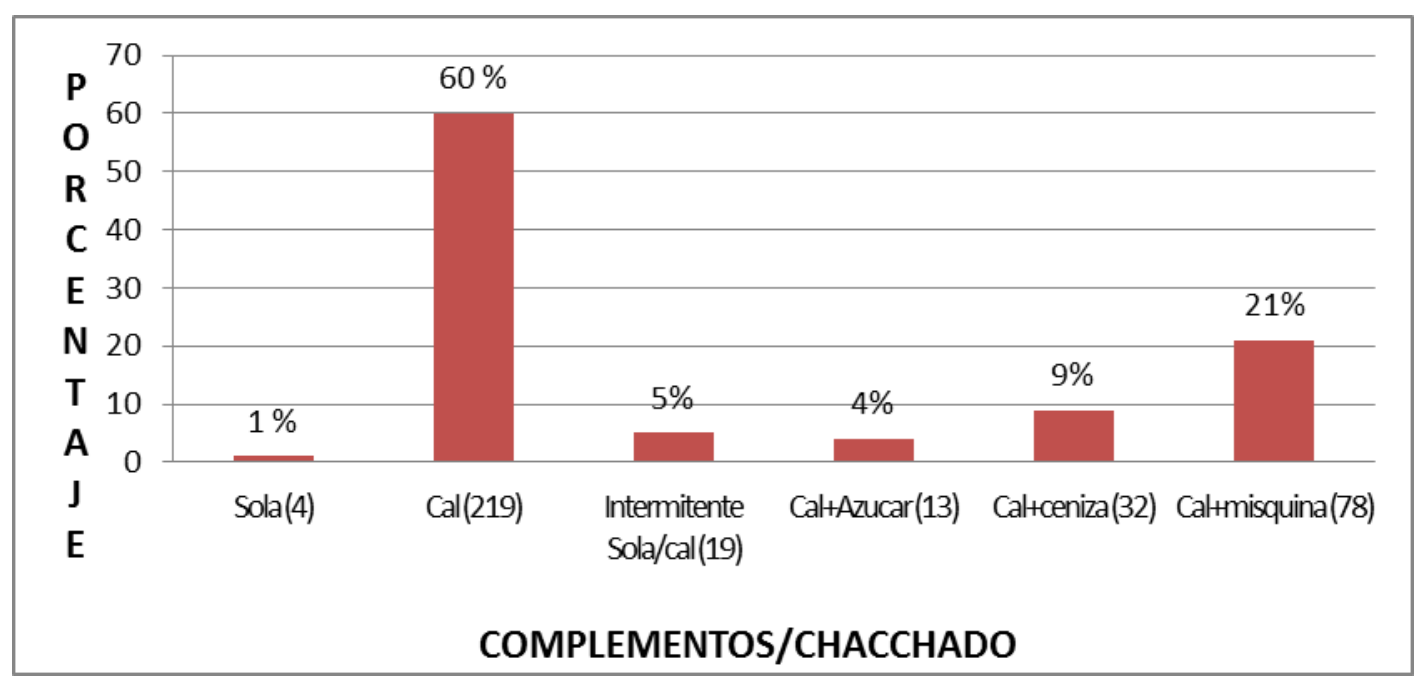

Figura 1. Complementos utilizados para el chacchado de hojas de coca por los integrantes de la muestra

Tabla 2. Distribución de piezas cariadas en el total de la muestra de acuerdo a grupo etáreo

EDAD EN AÑOS MUESTRA CARIADAS

$\begin{array}{lccc}20-29 & 79 & 330 & 23,7 \% \\ 30-39 & 117 & 481 & 34,5 \% \\ 40-49 & 70 & 262 & 18,8 \% \\ 50-60 & 99 & 320 & 23,0 \% \\ \text { TOTAL } & 365 & 1,393 & 100,0 \%\end{array}$

Tabla 3. Distribución de piezas perdidas en el total de la muestra de acuerdo a grupo etario 


\begin{tabular}{|c|c|c|c|}
\hline EDAD & MUESTRA & \multicolumn{2}{|c|}{ PERDIDAS } \\
\hline $20-29$ & 79 & 68 & $5,7 \%$ \\
\hline $30-39$ & 117 & 197 & $16,5 \%$ \\
\hline $40-49$ & 70 & 269 & $22,6 \%$ \\
\hline $50-60$ & 99 & 658 & $55,2 \%$ \\
\hline TOTAL & 365 & 1,192 & $100,0 \%$ \\
\hline
\end{tabular}

Tabla 4. Distribución de piezas obturadas en el total de la muestra de acuerdo a grupo etáreo

\begin{tabular}{cccc}
\hline EDAD & MUESTRA & \multicolumn{2}{l}{ OBTURADAS } \\
\hline $\mathbf{2 0 - 2 9}$ & 79 & 46 & $26,3 \%$ \\
\hline $\mathbf{3 0 - 3 9}$ & 117 & 69 & $39,4 \%$ \\
$\mathbf{4 0 - 4 9}$ & 70 & 26 & $14,9 \%$ \\
$\mathbf{5 0 - 6 0}$ & 99 & 34 & $19,4 \%$ \\
\hline
\end{tabular}


Tabla 5. Distribución de piezas cariadas con indicación de exodoncia en el total de la muestra de acuerdo a grupo etáreo

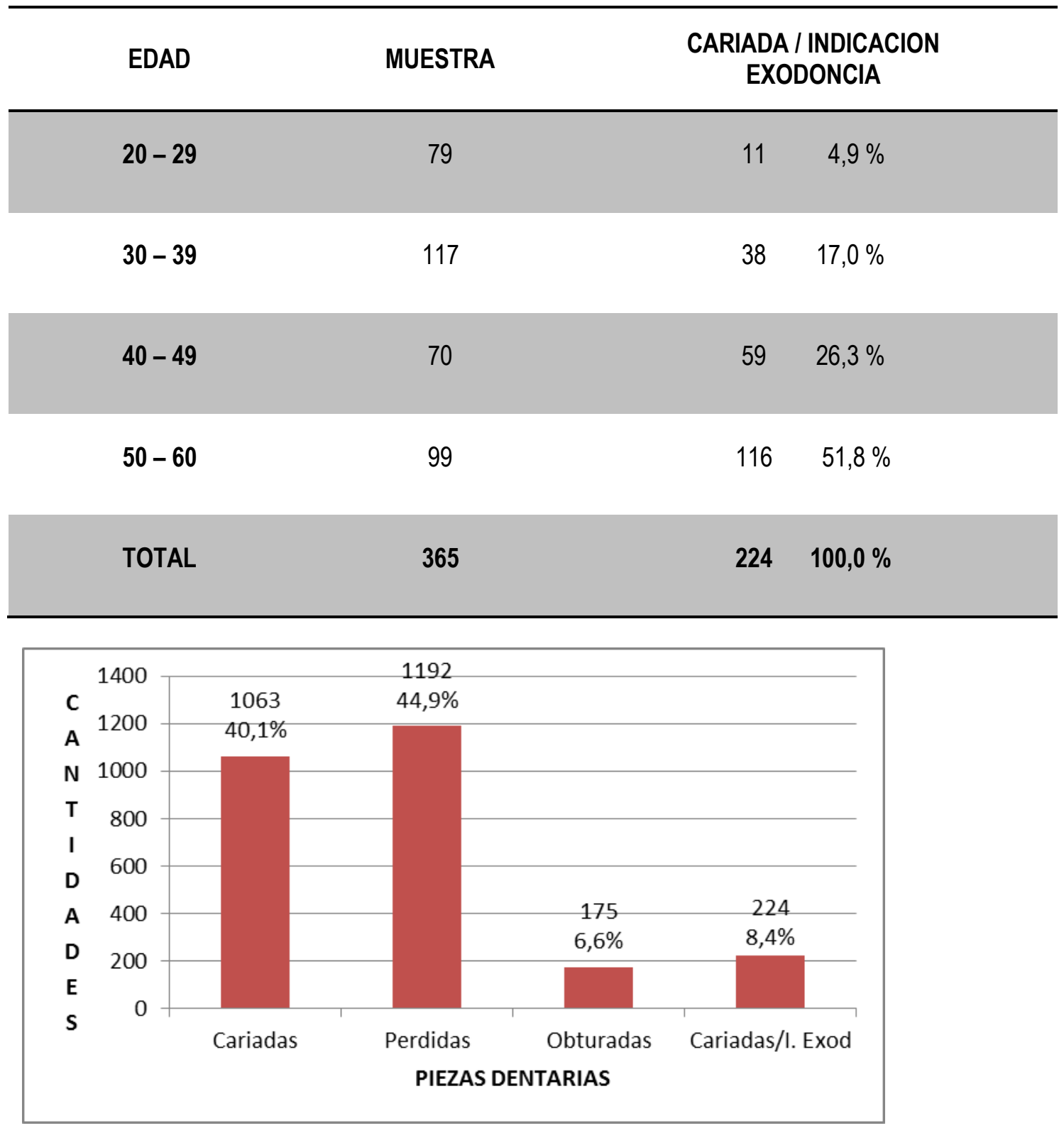

Figura 2. Distribución de piezas cariadas, perdidas, obturadas y con indicación de exodoncia en el total de la muestra.

Tabla 6. Índice CPOD de acuerdo a grupo etáreo y el total de la muestra

\begin{tabular}{cccccc}
\hline EDAD & $\mathbf{2 0 - 2 9}$ & $\mathbf{3 0 - 3 9}$ & $\mathbf{4 0 - 4 9}$ & $\mathbf{5 0 - 6 0}$ & $\begin{array}{c}\text { TOTAL DE LA } \\
\text { MUESTRA }\end{array}$ \\
\hline CPOD & 5,62 & 5,38 & 7,96 & 10,22 & 7,56 \\
\hline
\end{tabular}

